\title{
Semistar operations on Dedekind domains
}

\author{
Jesse Elliott \\ California State University, Channel Islands \\ One University Drive \\ Camarillo, CA 93012 USA \\ jesse.elliott@csuci.edu
}

November 20, 2018

\begin{abstract}
We give an explicit description of the lattice $\operatorname{Semistar}(D)$ of all semistar operations on any Dedekind domain $D$ from its set $\operatorname{Max}(D)$ of maximal ideals. This descpription is constructive if $\operatorname{Max}(D)$ is finite. As a corollary we show that $2^{\left(\begin{array}{c}n \\ {[n / 2]}\end{array}\right)} \leq|\operatorname{Semistar}(D)| \leq 2^{2^{n}}$ if $n=|\operatorname{Max}(D)|$ is finite; we compute $|\operatorname{Semistar}(D)|$ if $|\operatorname{Max}(D)| \leq 7$; and we show that if $\operatorname{Max}(D)$ is infinite then $\operatorname{Semistar}(D)$ has cardinality $2^{2^{|\operatorname{Max}(D)|}}$.
\end{abstract}

Keywords: semistar operation, Dedekind domain, principal ideal domain, closure operator, Moore family, multiplicative lattice

MSC: 13F05, 13F10, 13A15, 13G05, 06A15, 06F05

\section{Introduction}

Let $D$ be an integral domain with quotient field $K$. A semistar operation on $D$ is a closure operation $\star$ on the lattice $\mathcal{K}(D)$ of all nonzero $D$-submodules of $K$ such that $I^{\star} J^{\star} \subseteq(I J)^{\star}$ for all $I, J \in \mathcal{K}(D)$ [1] [3, Theorem 1.4]. Semistar operations were introduced in [11 as a generalization of star operations, which were introduced by Krull in [6, Section 6.43] in the guise of his '-operations.

An often-studied problem in the existing literature on semistar operations is to compute the cardinality of $\operatorname{Semistar}(D)$. It is clear that $D$ is a field if and only if $|\operatorname{Semistar}(D)|=1$, and it is well-known that $D$ is a discrete rank one valuation domain (DVR) if and only if $|\operatorname{Semistar}(D)|=2$ [11, Theorem 48]. One also has the following.

Theorem 1.1. Let $D$ be an integrally closed domain.

1. Semistar $(D)$ is finite if and only if $D$ is a finite dimensional Prüfer domain such that the set $\operatorname{Max}(D)$ of its maximal ideals is finite [7, (5.2)]. 
2. Suppose that $D$ is local and of finite dimension $n$. Then $|\operatorname{Semistar}(D)| \geq$ $n+1$, with equality holding if and only if $D$ is a discrete valuation domain (of rank $n$ ); and $D$ is a valuation domain if and only if $|\operatorname{Semistar}(D)| \leq$ $2 n+1[$, , Theorems 3 and 4].

Note that the set Semistar $(D)$ of all semistar operations on $D$ is not just a set: it is naturally partially ordered by the relation $\leq$, where $\star_{1} \leq \star_{2}$ if $I^{\star_{1}} \subseteq I^{\star_{2}}$ for all $I \in \mathcal{K}(D)$. The partially ordered set (poset) $\operatorname{Semistar}(D)$ is a complete lattice, and one has

$$
\begin{gathered}
I^{\wedge \Gamma}=\bigcap\left\{I^{\star}: \star \in \Gamma\right\}, \\
I^{\vee \Gamma}=\bigcap\left\{J \in \mathcal{K}(D): J \supseteq I \text { and } \forall \star \in \Gamma\left(J^{\star}=J\right)\right\},
\end{gathered}
$$

for all $\Gamma \subseteq \operatorname{Semistar}(D)$ and all $I \in \mathcal{K}(D)$ [3, Proposition 13.6]. In Theorem 3.4 and Corollary 3.5 we give an explicit description of the lattice $\operatorname{Semistar}(D)$ of all semistar operations on any Dedekind domain $D$ in terms of the set $\operatorname{Max}(D)$ of its maximal ideals.

For $\operatorname{Max}(D)$ finite our description of the lattice $\operatorname{Semistar}(D)$ is constructive and yields the following theorem. Let $J \in \mathcal{K}(D)$. For all $I \in \mathcal{K}(D)$ we let $I^{v(J)}=\left(J:_{K}\left(J:_{K} I\right)\right)$. The operation $v(J)$ on $\mathcal{K}(D)$ is a semistar operation on $D$ called divisorial closure on $D$ with respect to $J$ [12, Example 1.8(a)]. Note that $v(D)$ is the divisorial closure semistar operation $v: I \longmapsto\left(I^{-1}\right)^{-1}$ on $D$. Also, for any set $X$ we let $2^{X}$ denote the power set of $X$, partially ordered by inclusion.

Theorem 1.2. Let $D$ be a PID such that $\operatorname{Max}(D)$ is finite.

1. The lattice $\operatorname{Semistar}(D)$ is isomorphic to the lattice $\mathrm{C}\left(2^{\operatorname{Max}(D)}\right)$ of all closure operations on the complete lattice $2^{\operatorname{Max}(D)}$. Explicitly, the map

$$
\mathrm{C}\left(2^{\operatorname{Max}(D)}\right) \longrightarrow \text { Semistar }(D)
$$

acting by

$$
* \longmapsto \bigwedge\left\{v(I): I \in \mathcal{K}(D) \text { and }\left\{\mathfrak{p} \in \operatorname{Max}(D): I D_{\mathfrak{p}}=K\right\} \in\left(2^{\operatorname{Max}(D)}\right)^{*}\right\}
$$

is a poset isomorphism; alternatively, one has $* \longmapsto \star$, where $\star$ is the largest semistar operation on $D$ such that $I \in \mathcal{K}(D)$ is $\star$-closed if $\{\mathfrak{p} \in$ $\left.\operatorname{Max}(D): I D_{\mathfrak{p}}=K\right\}$ is $*$-closed in $2^{\operatorname{Max}(D)}$.

2. The lattice $\operatorname{Semistar}(D)$ is anti-isomorphic to the subposet

$\operatorname{Moore}\left(2^{\operatorname{Max}(D)}\right)=\left\{\mathcal{Y} \subseteq 2^{\operatorname{Max}(D)}: \mathcal{Y}\right.$ is closed under arbitrary intersections $\}$

of $2^{2^{\operatorname{Max}(D)}}$. Explicitly, the map

$$
\operatorname{Semistar}(D) \longrightarrow \operatorname{Moore}\left(2^{\operatorname{Max}(D)}\right)
$$


Table 1: Number of semistar operations on a Dedekind domain $D$

\begin{tabular}{c|c}
$|\operatorname{Max}(D)|$ & $|\operatorname{Semistar}(D)|$ \\
\hline 1 & 2 \\
2 & 7 \\
3 & 61 \\
4 & 2480 \\
5 & 1385552 \\
6 & 75973751474 \\
7 & 14087648235707352472
\end{tabular}

acting by

$$
\star \longmapsto\left\{\left\{\mathfrak{p} \in \operatorname{Max}(D): I^{\star} D_{\mathfrak{p}}=K\right\}: I \in \mathcal{K}(D)\right\}
$$

is a poset anti-isomorphism with inverse acting by

$$
\mathcal{Y} \longmapsto \bigwedge\left\{v(I): I \in \mathcal{K}(D) \text { and }\left\{\mathfrak{p} \in \operatorname{Max}(D): I D_{\mathfrak{p}}=K\right\} \in \mathcal{Y}\right\} .
$$

In Section 4 we obtain the following as a corollary of these constructions.

Theorem 1.3. Let $D$ and $D^{\prime}$ be Dedekind domains.

1. The lattices $\operatorname{Semistar}(D)$ and $\operatorname{Semistar}\left(D^{\prime}\right)$ are isomorphic if $\operatorname{Max}(D)$ and $\operatorname{Max}\left(D^{\prime}\right)$ have the same cardinality.

2. If $\operatorname{Max}(D)$ is infinite, then $|\operatorname{Semistar}(D)|$ is equal to $2^{2^{|\operatorname{Max}(D)|}}$.

3. If $|\operatorname{Max}(D)|=n$ is finite, then $2^{\left(\begin{array}{c}n \\ {[n / 2]}\end{array}\right)} \leq|\operatorname{Semistar}(D)| \leq 2^{2^{n}}$, and $|\operatorname{Semistar}(D)|$ is given for $n \leq 7$ as in Table 1 .

4. If $|\operatorname{Max}(D)|=2$, then $\operatorname{Semistar}(D)$ is isomorphic to $2^{\{1,2,3\}}-\{\{1\}\}$.

5. If $|\operatorname{Max}(D)|=3$, then $\operatorname{Semistar}(D)$ is anti-isomorphic to the lattice with Hasse diagram given as in [4, Figure 1].

In the spirit of Theorem 1.1 we make the following conjecture.

Conjecture 1.4. Let $D$ be an integrally closed domain such that $\operatorname{Max}(D)$ is finite. Then $D$ is a principal ideal domain (PID) if and only if $|\operatorname{Semistar}(D)|$ is equal to the number of subsets of $2^{\operatorname{Max}(D)}$ that are closed under arbitrary intersections.

\section{Background on semistar operations and nuclei}

A magma is a set $M$ equipped with a binary operation on $M$ (which we write multiplicatively). An ordered magma is a magma $M$ equipped with a partial ordering $\leq$ such that $x \leq y$ and $x^{\prime} \leq y^{\prime}$ imply $x x^{\prime} \leq y y^{\prime}$ for all $x, y, x^{\prime}, y^{\prime} \in M$. An 
ordered magma $M$ is said to be a multiplicative lattice (resp., near multiplicative lattice) if $M$ is a commutative monoid and one has $\bigvee(X Y)=(\bigvee X)(\bigvee Y)$ for all subsets (resp., nonempty subsets) $X, Y$ of $M$ [3]. A multiplicative lattice is equivalently a near multiplicative lattice $M$ such that $\bigwedge M$ exists and annihilates every element of $M$. For the purposes of this paper the relevant examples are as follows.

Example 2.1. Let $D$ be an integral domain with quotient field $K$.

1. The complete lattice $\operatorname{Mod}_{D}(K)$ of all $D$-submodules of $K$ is a multiplicative lattice under the operation $(I, J) \longmapsto I J$.

2. The lattice $\mathcal{K}(D)$ of all nonzero $D$-submodules of $K$ is a near multiplicative lattice.

For any self-map $\star$ of a set $X$ we write $x^{\star}=\star(x)$ for all $x \in X$. A closure operation on a poset $S$ is a self-map $\star$ of $S$ satisfying the following conditions for all $x, y \in S$.

1. $x \leq x^{\star}$.

2. $x \leq y$ implies $x^{\star} \leq y^{\star}$.

3. $\left(x^{\star}\right)^{\star}=x^{\star}$.

A nucleus on an ordered magma $M$ is a closure operation $\star$ on $M$ such that $x^{\star} y^{\star} \leq(x y)^{\star}$ for all $x, y \in M$. Nuclei were first studied in the contexts of ideal lattices and locales and later in the context of quantales as quantic nuclei [10].

Theorem 2.2 ([3, Theorem 1.4]). A semistar operation on an integral domain $D$ is equivalently a nucleus on the near multiplicative lattice $\mathcal{K}(D)$. Moreover, the following are equivalent for any self-map $\star$ of $\mathcal{K}(D)$.

1. $\star$ is a semistar operation on $D$.

2. $\star$ is a closure operation on the lattice $\mathcal{K}(D)$ and $(a I)^{\star}=a I^{\star}$ for all $I \in$ $\mathcal{K}(D)$ and all nonzero $a \in D$.

3. $\star$ is a closure operation on the lattice $\mathcal{K}(D)$ and $\left(I^{\star} J^{\star}\right)^{\star}=(I J)^{\star}$ for all $I, J \in \mathcal{K}(D)$.

4. $I J \subseteq K^{\star}$ if and only if $I J^{\star} \subseteq K^{\star}$ for all $I, J, K \in \mathcal{K}(D)$.

If $\star_{1}$ and $\star_{2}$ are nuclei on an ordered magma $M$, then we write $\star_{1} \leq \star_{2}$ if $x^{\star_{1}} \leq x^{\star_{2}}$ for all $x \in M$. This defines a partial ordering on the set $\mathrm{N}(M)$ of all nuclei on $M$. If $\bigvee X$ exists in $M$ for all nonempty subsets $X$ of $M$ (which holds if $M$ is a near multiplicative lattice), then by [3, Proposition 4.3] the poset $\mathrm{N}(M)$ is a complete lattice and one has

$$
\begin{gathered}
x^{\wedge \Gamma}=\bigwedge\left\{x^{\star}: \star \in \Gamma\right\}, \\
x^{\vee \Gamma}=\bigwedge\left\{y \in M: y \geq x \text { and } \forall \star \in \Gamma\left(y^{\star}=y\right)\right\},
\end{gathered}
$$

for all $\Gamma \subseteq \mathrm{N}(M)$ and all $x \in M$. 
Corollary 2.3. For any integral domain $D$ one has $\operatorname{Semistar}(D)=\mathrm{N}(\mathcal{K}(D))$ as partially ordered sets.

If $M$ is an ordered magma, then the map $\mathrm{N}(M) \longrightarrow 2^{M}$ acting by $\star \longmapsto M^{\star}$ is an anti-embedding of $\mathrm{N}(M)$ in $2^{M}$. We will denote the image $\left\{M^{\star}: \star \epsilon\right.$ $\mathrm{N}(M)$ \} of this anti-embedding by $\mathrm{M}(M)$. In particular, the poset $\mathrm{N}(M)$ is anti-isomorphic to the poset $\mathrm{M}(M)$. [3, Proposition 4.6] implies the following.

Proposition 2.4. Let $D$ be an integral domain with quotient field $K$. The poset $\mathrm{M}(\mathcal{K}(D)$ ) consists of all sets of the form $\mathcal{C}-\{(0)\}$, where $\mathcal{C}$ is any subset of $\mathcal{K}(D)$ satisfying the following three condtions: (1) (0) $\in \mathcal{C}$; (2) if $\mathcal{I} \subseteq \mathcal{C}$ then $\bigcap \mathcal{I} \in \mathcal{C}$; and (3) if $I \in \mathcal{C}$ then $\left(I:_{K} J\right) \in \mathcal{C}$ for all $J \in \mathcal{K}(D)$. Moreover, the complete lattice $\operatorname{Semistar}(D)=\mathrm{N}(\mathcal{K}(D))$ of all semistar operations on $D$ is anti-isomorphic to the poset $\mathrm{M}(\mathcal{K}(D))$.

\section{Constructing the lattice of all semistar oper- ations}

In this section we construct the lattice $\operatorname{Semistar}(D)=\mathrm{N}(\mathcal{K}(D))$ of all semistar operations on any Dedekind domain $D$ from the set $\operatorname{Max}(D)$ of its maximal ideals.

If $D$ is a Dedekind domain, then for any $\mathfrak{p} \in \operatorname{Max}(D)$ we let $v_{\mathfrak{p}}: K \longrightarrow$ $\mathbb{Z} \cup\{\infty\}$ denote the normalized $\mathfrak{p}$-adic valuation on $K$. We let $\mathbb{Z}[\infty]$ denote the near multiplicative lattice $\mathbb{Z} \cup\{\infty\}$, where $a \leq \infty$ for all $a \in \mathbb{Z}$ and $a+\infty=\infty=$ $\infty+a$ for all $a \in \mathbb{Z}[\infty]$. Also, we let $\mathbb{Z}[ \pm \infty]$ denote the multiplicative lattice obtained from $\mathbb{Z}[\infty]$ by adjoining an annihilator $-\infty=\inf \mathbb{Z}[\infty]$. In particular, we assume $-\infty+\infty=-\infty=\infty+(-\infty)$. Also, for any $x \in \mathbb{Z}[ \pm \infty]$ we define $-x$ and $|x|$ in $\mathbb{Z}[ \pm \infty]$ in the obvious way.

For any near multiplicative lattice $M$ and any set $S$ the set $M^{S}$ of all functions from $S$ to $M$ is a near multiplicative lattice under pointwise multiplication. For any set $S$ we let

$$
\mathcal{Z}(S)=\left\{f: S \longrightarrow \mathbb{Z}[\infty]: f^{-1}\left(\mathbb{Z}_{<0}\right) \text { is finite }\right\},
$$

which is a sub near multiplicative lattice of the multiplicative lattice $\mathbb{Z}[ \pm \infty]^{S}$.

In the next proposition we show that $\mathcal{K}(D) \cong \mathcal{Z}(\operatorname{Max}(D))$ as near multiplicative lattices. Let $\mathfrak{p} \in \operatorname{Max}(D)$. Define $\mathfrak{p}^{\infty} D_{\mathfrak{p}}=(0)$ and $\mathfrak{p}^{-\infty} D_{\mathfrak{p}}=K$. For any $I \in \mathcal{K}(D)$ we define $v_{\mathfrak{p}}(I) \in \mathbb{Z} \cup\{ \pm \infty\}$ so that $I D_{\mathfrak{p}}=\mathfrak{p}^{v_{\mathfrak{p}}(I)} D_{\mathfrak{p}}$. For any function $f: \operatorname{Max}(D) \longrightarrow \mathbb{Z}[ \pm \infty]$ we let

$$
[f]=\bigcap_{\mathfrak{p} \in \operatorname{Max}(D)} \mathfrak{p}^{-f(\mathfrak{p})} D_{\mathfrak{p}}=\left\{x \in K: v_{\mathfrak{p}}(x) \geq-f(\mathfrak{p}) \text { for all } \mathfrak{p} \in \operatorname{Max}(D)\right\} .
$$

There is a map $[-]: \mathbb{Z}[ \pm \infty]^{\operatorname{Max}(D)} \longrightarrow \operatorname{Mod}_{D}(K)$ acting by $f \longmapsto[f]$.

Proposition 3.1. Let $D$ be a Dedekind domain, and let $f, g \in \mathbb{Z}[ \pm \infty]^{\operatorname{Max}(D)}$. 
1. $f \in \mathcal{Z}(\operatorname{Max}(D))$ if and only if $[f] \neq(0)$.

2. If $f \in \mathcal{Z}(\operatorname{Max}(D))$, then $[f] D_{\mathfrak{p}}=\mathfrak{p}^{-f(\mathfrak{p})} D_{\mathfrak{p}}$ for all $\mathfrak{p} \in \operatorname{Max}(D)$.

3. If $f, g \in \mathcal{Z}(\operatorname{Max}(D))$, then $\left([f]:_{K}[g]\right)=[-(-f+g)]$.

4. The map $[-]: \mathcal{Z}(\operatorname{Max}(D)) \longrightarrow \mathcal{K}(D)$ is an isomorphism of near multiplicative lattices with inverse acting by $I \longmapsto\left(\mathfrak{p} \longmapsto-v_{\mathfrak{p}}(I)\right)$.

Proof. Statement (1) is clear. We prove statement (2). Clearly one has $[f] D_{\mathfrak{p}} \subseteq$ $\mathfrak{p}^{-f(\mathfrak{p})} D_{\mathfrak{p}}$. We wish to show the reverse containment. Let $x \in \mathfrak{p}^{-f(\mathfrak{p})} D_{\mathfrak{p}}$. By the weak approximation theorem for Dedekind domains, there exists $y \in K$ such that

$$
v_{\mathfrak{q}}(y)= \begin{cases}v_{\mathfrak{q}}(x) & \text { if } \mathfrak{q}=\mathfrak{p} \\ -f(\mathfrak{q}) & \text { if } \mathfrak{q} \neq \mathfrak{p} \text { and } f(\mathfrak{q})<0 \\ \geq 0 & \text { otherwise. }\end{cases}
$$

Thus $x D_{\mathfrak{p}}=y D_{\mathfrak{p}}$ and $y \in[f]$, whence $x \in[f] D_{\mathfrak{p}}$. This proves (2). To prove statement (3), note that

$$
\begin{aligned}
\left([f]:_{K}[g]\right) & =\bigcap_{\mathfrak{p}}\left(\mathfrak{p}^{-f(\mathfrak{p})} D_{\mathfrak{p}}:_{K}[g]\right) \\
& =\bigcap_{f(\mathfrak{p})<\infty}\left(\mathfrak{p}^{-f(\mathfrak{p})}\left(D_{\mathfrak{p}}:_{K}[g] D_{\mathfrak{p}}\right)\right) \\
& =\bigcap_{f(\mathfrak{p})<\infty}\left(\mathfrak{p}^{-f(\mathfrak{p})}\left(D_{\mathfrak{p}}:_{K} \mathfrak{p}^{-g(\mathfrak{p})} D_{\mathfrak{p}}\right)\right) \\
& =\bigcap_{f(\mathfrak{p})<\infty} \mathfrak{p}^{-f(\mathfrak{p})+g(\mathfrak{p})} D_{\mathfrak{p}} \\
& =[-(-f+g)] .
\end{aligned}
$$

Finally, statement (4) follows readily from statement (3) and the fact that $I=$ $\bigcap_{\mathfrak{p} \in \operatorname{Max}(D)} \mathfrak{p}^{v_{\mathfrak{p}}(I)} D_{\mathfrak{p}}$ and $v_{\mathfrak{p}}(I J)=v_{\mathfrak{p}}(I)+v_{\mathfrak{p}}(J)$ for all $I, J \in \mathcal{K}(D)$.

For any set $S$ and any $f, g \in \mathbb{Z}[ \pm \infty]^{S}$, we write $f \preceq g$ if $f(x) \leq g(x)$ for almost all $x \in S$ and $f(x)=\infty$ if and only if $g(x)=\infty$ for all $x \in S$. The relation $\preceq$ is a preorder on $\mathbb{Z}[ \pm \infty]^{S}$.

Lemma 3.2. Let $S$ be a set and $\mathcal{X} \subseteq \mathcal{Z}(S)$. Consider the following conditions on $\mathcal{X}$.

1. If $\mathcal{Y} \subseteq \mathcal{X}$ and $\inf \mathcal{Y} \in \mathcal{Z}(S)$, then $\inf \mathcal{Y} \in \mathcal{X}$.

2. If $f \in \mathcal{X}$ and $g \in \mathcal{Z}(S)$ with $g \preceq f$, then $g \in \mathcal{X}$.

3. If $f \in \mathcal{X}$ and $g,-(-f+g) \in \mathcal{Z}(S)$ then $-(-f+g) \in \mathcal{X}$.

One has $(1) \wedge(2) \Leftrightarrow(1) \wedge(3)$ and $(2) \Rightarrow(3)$. Moreover, if $S=\operatorname{Max}(D)$, where $D$ is a Dedekind domain, and if $[\mathcal{X}]$ for any $\mathcal{X} \subseteq \mathcal{Z}(\operatorname{Max}(D))$ denotes the image of $\mathcal{X}$ under the isomorphism $[-]: \mathcal{Z}(\operatorname{Max}(D)) \longrightarrow \mathcal{K}(D)$, then the above conditions hold if and only if $[\mathcal{X}]=\mathcal{K}(D)^{\star}$ for some semistar operation $\star$ on $D$. 
Proof. First, suppose that condition (2) holds. Let $f \in \mathcal{X}$ and $g \in \mathcal{Z}(S)$ with $h=-(-f+g) \in \mathcal{Z}(S)$. Note that $h(x)=\infty$ if $f(x)=\infty$, and if $f(x)<\infty$ then $g(x)<\infty$ and $h(x)=f(x)-g(x)<\infty$. In particular, $h(x)>f(x)$ implies $g(x)<0$, which holds for only finitely many $x$. It follows, then, that $h \preceq f$, whence $h=-(-f+g) \in \mathcal{Z}(S)$. This shows that $(2) \Rightarrow(3)$.

Next, suppose $(1) \wedge(3)$ holds. Let $f \in \mathcal{X}$ and $g \in \mathcal{Z}(S)$ with $g \preceq f$. Let $x \in S$ with $g(x)<\infty$ (so $f(x)<\infty$ ). Define $h_{x} \in \mathcal{Z}(S)$ as follows:

$$
h_{x}(y)= \begin{cases}f(y)-g(y) & \text { if } y=x \text { or if } y \neq x \text { and } g(y)>f(y) \\ 0 & \text { otherwise. }\end{cases}
$$

Then

$$
-\left(-f+h_{x}\right)(y)= \begin{cases}g(y) & \text { if } y=x \text { or if } y \neq x \text { and } g(y)>f(y) \\ f(y) & \text { otherwise. }\end{cases}
$$

By condition (3) we have $-\left(-f+h_{x}\right) \in \mathcal{X}$. Therefore, by condition (1), we have $g=\inf _{g(x)<\infty}-\left(-f+h_{x}\right) \in \mathcal{X}$. Thus $(1) \wedge(3) \Rightarrow(2)$, and since $(2) \Rightarrow(3)$ we have $(1) \wedge(2) \Leftrightarrow(1) \wedge(3)$.

Finally, if $S=\operatorname{Max}(D)$, where $D$ is a Dedekind domain, then, by Propositions 2.4 and 3.1. one has $[\mathcal{X}]=\mathcal{K}(D)^{\star}$ for some semistar operation $\star$ on $D$ if and only if conditions (1) and (3) hold.

Lemma 3.3. Let $S$ be a set. For any $\mathcal{X} \subseteq \mathcal{Z}(S)$, let

$$
\mathcal{X} \preceq=\{g \in \mathcal{Z}(S): g \preceq f \text { for some } f \in \mathcal{X}\},
$$

and let

$$
\mathcal{X}^{\inf }=\{\inf \mathcal{Y}: \mathcal{Y} \subseteq \mathcal{X} \text { and } \inf \mathcal{Y} \in \mathcal{Z}(S)\} .
$$

1. $\mathcal{X} \longmapsto \mathcal{X} \preceq$ and $\mathcal{X} \longmapsto \mathcal{X}^{\text {inf }}$ are closure operations on $2^{\mathcal{Z}(S)}$.

2. For any $\mathcal{X} \subseteq \mathcal{Z}(S)$ one has $(\mathcal{X} \preceq)^{\inf } \supseteq\left(\mathcal{X}^{\inf }\right) \preceq$.

3. $\mathcal{X}^{\dagger}=(\mathcal{X} \preceq)^{\mathrm{inf}}$ is the smallest subset $\mathcal{W}$ of $\mathcal{Z}(S)$ containing $\mathcal{X}$ such that $\mathcal{W} \preceq \subseteq \mathcal{W}$ and $\mathcal{W}^{\inf } \subseteq \mathcal{W}$.

4. $\mathcal{X} \longmapsto \mathcal{X}^{\dagger}$ is a closure operation on $2^{\mathcal{Z}(S)}$.

Proof. Statement (1) is clear. To prove (2), let $g \in\left(\mathcal{X}^{\inf }\right) \preceq$. Then $g \preceq \inf _{\lambda} f_{\lambda}$, where $f_{\lambda} \in \mathcal{X}$ for all $\lambda$ in some indexing set and $\inf _{\lambda} f_{\lambda} \in \mathcal{Z}(S)$. Now, for almost all $x \in S$ one has $g(x) \leq f_{\lambda}(x)$ for all $\lambda$, and also $g(x)=\infty$ if and only if $f_{\lambda}(x)=\infty$ for all $\lambda$. Let

$$
g_{\lambda}(x)= \begin{cases}g(x) & \text { if } f_{\lambda}(x)<\infty \\ \infty & \text { otherwise }\end{cases}
$$

Since $g \in \mathcal{Z}(S)$ we have $g_{\lambda} \in \mathcal{Z}(S)$ for all $\lambda$. Moreover, one has $g_{\lambda} \preceq f_{\lambda}$, and therefore $g_{\lambda} \in \mathcal{X} \preceq$, for all $\lambda$. Finally, since $g=\inf _{\lambda} g_{\lambda}$, we have $g \in(\mathcal{X} \preceq)^{\text {inf }}$. This proves (2). Finally, statements (3) and (4) follow readily from statement (2) and [3, Proposition 4.8]. 
Combining Proposition 3.1 with Lemmas 3.2 and 3.3 we obtain the following.

Theorem 3.4. Let $D$ be a Dedekind domain. For any $\mathcal{X} \subseteq \mathcal{Z}(\operatorname{Max}(D))$, let

$$
\mathcal{X} \preceq=\{g \in \mathcal{Z}(\operatorname{Max}(D)): g \preceq f \text { for some } f \in \mathcal{X}\},
$$

and let

$$
\mathcal{X}^{\inf }=\{\inf \mathcal{Y}: \mathcal{Y} \subseteq \mathcal{X} \text { and } \inf \mathcal{Y} \in \mathcal{Z}(\operatorname{Max}(D))\} .
$$

Let $[\mathcal{X}]$ denote the image of $\mathcal{X}$ under the isomorphism $[-]: \mathcal{Z}(\operatorname{Max}(D)) \longrightarrow$ $\mathcal{K}(D)$ of Proposition 3.1.

1. $\mathcal{X}^{\dagger}=(\mathcal{X} \preceq)^{\inf }$ is the smallest subset of $\mathcal{Z}(\operatorname{Max}(D))$ containing $\mathcal{X}$ such that $\left[\mathcal{X}^{\dagger}\right]=\mathcal{K}(D)^{\star}$ for some semistar operation $\star$ on $D$.

2. The self-map of $\dagger$ of $2^{\mathcal{Z}(\operatorname{Max}(D))}$ acting by $\mathcal{X} \longmapsto \mathcal{X}^{\dagger}$ is a closure operation on $2^{\mathcal{Z}(\operatorname{Max}(D))}$.

3. $\mathcal{X} \in\left(2^{\mathcal{Z}(\operatorname{Max}(D))}\right)^{\dagger}$ if and only if $\mathcal{X} \preceq \subseteq \mathcal{X}$ and $\mathcal{X}^{\text {inf }} \subseteq \mathcal{X}$.

4. The map $\left(2^{\mathcal{Z}(\operatorname{Max}(D))}\right)^{\dagger} \longrightarrow \mathrm{M}(\mathcal{K}(D))$ acting by $\mathcal{X} \longmapsto[\mathcal{X}]$ is an isomorphism of posets; explicitly, this isomorphism acts by

$$
\mathcal{X} \longmapsto\left\{\bigcap_{\mathfrak{p} \in \operatorname{Max}(D)} \mathfrak{p}^{-f(\mathfrak{p})} D_{\mathfrak{p}}: f \in \mathcal{X}\right\}
$$

with inverse acting by

$$
\mathcal{C} \longmapsto\left\{\mathfrak{p} \longmapsto-v_{\mathfrak{p}}(I): I \in \mathcal{C}\right\} .
$$

By [3, Proposition 13.8] the inverse of the poset anti-isomorphism Semistar $(D) \longrightarrow$ $\mathrm{M}(\mathcal{K}(D))$ acting by $\star \longmapsto \mathcal{K}(D)^{\star}$ acts by $\mathcal{C} \longmapsto \bigwedge\{v(J): J \in \mathcal{C}\}$. Thus we have the following.

Corollary 3.5. For any Dedekind domain D, the poset Semistar $(D)$ of all semistar operations on $D$ is anti-isomorphic to the subposet

$$
\begin{aligned}
\left(2^{\mathcal{Z}(\operatorname{Max}(D))}\right)^{\dagger} & =\left\{(\mathcal{X} \preceq)^{\mathrm{inf}}: \mathcal{X} \in 2^{\mathcal{Z}(\operatorname{Max}(D))}\right\} \\
& =\left\{\mathcal{X} \in 2^{\mathcal{Z}(\operatorname{Max}(D))}: \mathcal{X} \preceq \subseteq \mathcal{X} \text { and } \mathcal{X}^{\text {inf }} \subseteq \mathcal{X}\right\}
\end{aligned}
$$

of $2^{\mathcal{Z}(\operatorname{Max}(D))}$. Explicitly, there is a poset anti-isomorphism

$$
\operatorname{Semistar}(D) \longrightarrow\left(2^{\mathcal{Z}(\operatorname{Max}(D))}\right)^{\dagger}
$$

acting by

$$
\star \longmapsto\left\{\mathfrak{p} \longmapsto-v_{\mathfrak{p}}(I): I \in \mathcal{K}(D)^{\star}\right\}
$$

with inverse acting by

$$
\mathcal{X} \longmapsto \bigwedge\left\{v\left(\bigcap_{\mathfrak{p} \in \operatorname{Max}(D)} \mathfrak{p}^{-f(\mathfrak{p})} D_{\mathfrak{p}}\right): f \in \mathcal{X}\right\} .
$$




\section{Consequences of the construction}

The results in this section yield Theorem 1.2 and 1.3 of the introduction.

Let $X$ be a poset. The set $\mathrm{C}(X)$ of closure operations on $X$ is partially ordered by the obvious relation. The map $\mathrm{C}(X) \longrightarrow 2^{X}$ acting by $\star \longmapsto X^{\star}$ is an anti-embedding of $\mathrm{C}(X)$ in $2^{X}$. We will denote the image $\left\{X^{\star}: \star \in \mathrm{C}(X)\right\}$ of this anti-embedding by $\operatorname{Moore}(X)$. In particular, the poset $\mathrm{C}(X)$ is antiisomorphic to the poset $\operatorname{Moore}(X)$.

Let $S$ be a set. By [3, Corollary 4.7(1)], one has

$$
\text { Moore }\left(2^{S}\right)=\left\{\mathcal{Y} \subseteq 2^{S}: \mathcal{Y} \text { is closed under arbitrary intersections }\right\} .
$$

(Moore $\left(2^{S}\right)$ is the set of all Moore families on $S$ [2].) For any $X \subseteq S$, define $\iota_{X} \in \mathcal{Z}(S)$ as follows:

$$
\iota_{X}(x)= \begin{cases}\infty & \text { if } x \in X \\ 0 & \text { otherwise }\end{cases}
$$

Then

$\left\{\iota_{X}\right\}^{\dagger}=\{f \in \mathcal{Z}(S): f(x)=\infty$ iff $x \in X$, and $f(x)=0$ for almost all $x \in S-X\}$.

We let $\iota(X)=\left\{\iota_{X}\right\}^{\dagger}$, and for any $\mathcal{Y} \subseteq 2^{S}$ we let

$$
\begin{aligned}
\iota(\mathcal{Y}) & =\bigcup_{X \in \mathcal{Y}} \iota(X) \\
& =\left\{f \in \mathcal{Z}(S): f^{-1}(\infty) \in \mathcal{Y} \text { and } f(x)=0 \text { for almost all } x \in S-f^{-1}(\infty)\right\} .
\end{aligned}
$$

Lemma 4.1. Let $S$ be a set.

1. The map $\iota: 2^{2^{S}} \longrightarrow 2^{\mathcal{Z}(S)}$ is an embedding of posets and has an orderpreserving left inverse acting by $\mathcal{X} \longmapsto\left\{f^{-1}(\infty): f \in \mathcal{X}\right\}$.

2. The map $\iota:$ Moore $\left(2^{S}\right) \longrightarrow\left(2^{\mathcal{Z}(S)}\right)^{\dagger}$ is an embedding of posets and has an order-preserving left inverse acting by $\mathcal{X} \longmapsto\left\{f^{-1}(\infty): f \in \mathcal{X}\right\}$.

3. If $S$ is finite, then the map $\iota: \operatorname{Moore}\left(2^{S}\right) \longrightarrow\left(2^{\mathcal{Z}(S)}\right)^{\dagger}$ is a poset isomorphism, and one has $\iota(\mathcal{Y})=\left\{f \in \mathcal{Z}(S): f^{-1}(\infty) \in \mathcal{Y}\right\}$ for all $\mathcal{Y} \in \operatorname{Moore}\left(2^{S}\right)$.

Proof. Statement (1) is clear. To prove (2), first note that if $f \in \iota(X)$ and $g \preceq f$, where $X \subseteq S$ and $g \in \mathcal{Z}(S)$, then $g \in \iota(X)$; and if $f_{\lambda} \in \iota\left(X_{\lambda}\right)$ for all $\lambda$ in some indexing set, where each $X_{\lambda} \subseteq S$ and $\inf _{\lambda} f_{\lambda} \in \mathcal{Z}(S)$, then $\inf _{\lambda} f_{\lambda} \in$ $\iota\left(\bigcap_{\lambda} X_{\lambda}\right)$. This shows that $\iota$ maps $\operatorname{Moore}\left(2^{S}\right)$ into $\left(2^{\mathcal{Z}(S)}\right)^{\dagger}$. Next, if $\mathcal{X} \in$ $\left(2^{\mathcal{Z}(S)}\right)^{\dagger}$ and $f \in \mathcal{X}$, then $\iota_{f^{-1}(\infty)} \preceq f$, whence $\iota_{f^{-1}(\infty)} \in \mathcal{X}$. Therefore, if $\mathcal{Y} \subseteq$ $\mathcal{X}$, then $\inf _{f \in \mathcal{Y}} \iota_{f}^{-1}(\infty)=\iota_{Y} \in \mathcal{X}$, where $Y=\bigcap_{f \in \mathcal{Y}} f^{-1}(\infty)$, so $\bigcap_{f \in \mathcal{Y}} f^{-1}(\infty) \in$ $\left\{f^{-1}(\infty): f \in \mathcal{X}\right\}$. This shows that the left inverse of $\iota$ maps $\left(2^{\mathcal{Z}(S)}\right)^{\dagger}$ into Moore $\left(2^{S}\right)$ and therefore completes the proof of statement (2). Finally, suppose that $S$ is finite. Then for any $f \in \mathcal{Z}(S)$ one has $f \in \iota\left(f^{-1}(\infty)\right)$. Therefore, if $\mathcal{X} \in\left(2^{\mathcal{Z}(S)}\right)^{\dagger}$, then $\mathcal{X}=\bigcup_{f \in \mathcal{X}} \iota\left(f^{-1}(\infty)\right)=\iota\left(\left\{f^{-1}(\infty): f \in \mathcal{X}\right\}\right)$, which proves that the two maps in statement (2) are inverses of each other in this case. 
Combining Lemma 4.1 above with Theorem 3.4 and Corollary 3.5, we obtain Theorem 1.2 of the introduction. We also have the following.

Corollary 4.2. Let $D$ be a Dedekind domain. If $\operatorname{Max}(D)$ is infinite, then the set $\operatorname{Semistar}(D)$ of all semistar operations on $D$ has cardinality $2^{2^{|\operatorname{Max}(D)|}}$.

Proof. Note first that $\mathcal{Z}(\operatorname{Max}(D))$ has cardinality $\aleph_{0}^{|\operatorname{Max}(D)|}=2^{|\operatorname{Max}(D)|}$. Therefore, by Corollary 3.5 the set $\operatorname{Semistar}(D)$ has cardinality at most $2^{2^{|\operatorname{Max}(D)|}}$. On the other hand, by Theorem 3.4(4) and Lemma 4.1(2), the set Semistar $(D)$ has cardinality at least $\left|\operatorname{Moore}\left(2^{\operatorname{Max}(D)}\right)\right|$. Now, Moore $\left(2^{S}\right)$ for any set $S$ is isomorphic to the poset

$$
\mathcal{U}\left(2^{S}\right)=\left\{\mathcal{Y} \subseteq 2^{S}: \mathcal{Y} \text { is closed under arbitrary unions }\right\} .
$$

Moreover, the poset $\mathcal{U}\left(2^{S}\right)$ contains the set of all ultrafilters on the set $S$, which for any infinite set $S$ is known to have cardinality $2^{2^{|S|}}$. Therefore $|\operatorname{Semistar}(D)| \geq\left|\operatorname{Moore}\left(2^{\operatorname{Max}(D)}\right)\right|=\left|\mathcal{U}\left(2^{\operatorname{Max}(D)}\right)\right| \geq 2^{2^{|\operatorname{Max}(D)|}}$, whence equalities hold.

Corollary 4.3. The set of all semistar operations on $\mathbb{Z}$ has cardinality $2^{2^{\aleph_{0}}}$.

Given also [2, Table 1] and [4, Figure 1], we obtain the following.

Corollary 4.4. Let $D$ be a PID such that $\operatorname{Max}(D)$ is finite.

1. Semistar $(D)$ is finite and has cardinality given as in Table 1 for small values of $|\operatorname{Max}(D)|$.

2. If $|\operatorname{Max}(D)|=2$, then $\operatorname{Semistar}(D) \cong 2^{\{1,2,3\}}-\{\{1\}\}$.

3. If $|\operatorname{Max}(D)|=3$, then $\operatorname{Semistar}(D)$ is anti-isomorphic to the lattice with Hasse diagram given as in [4, Figure 1].

It is known that, if $X_{n}$ is a set of cardinality $n$ for every positive integer $n$, then

$$
\left|\operatorname{Moore}\left(2^{X_{n}}\right)\right|=2^{\left(\begin{array}{c}
n \\
{[n / 2]}
\end{array}\right) f(n)},
$$

where $f(n) \geq 1$ for all $n$ and $f(n)=1+O\left(n^{-1 / 4} \log _{2} n\right)$ as $n \longrightarrow \infty$ and therefore $\lim _{n \rightarrow \infty} f(n)=1$ [1, Theorem 1]. Thus we have the following.

Corollary 4.5. Let $D$ be a Dedekind domain with quotient field $K$, and let $K \supsetneq D_{1} \supsetneq D_{2} \supsetneq D_{3} \supsetneq \cdots$ be an infinite descending chain of overrings of $D$ such that $m_{n}=\left|\operatorname{Max}\left(D_{n}\right)\right|<\infty$ for all $n$. Then we have the following.

1. There is a unique strictly ascending sequence $X_{1} \subsetneq X_{2} \subsetneq X_{2} \subsetneq \cdots$ of subsets of $\operatorname{Max}(D)$ such that $D_{n}=U_{n}^{-1} D$, where $U_{n}=D-\bigcup X_{n}$.

2. One has $m_{n}=\left|X_{n}\right|$ for all $n$, and therefore $m_{n}$ is a strictly increasing sequence of positive integers. 
3. If $s_{n}=\left|\operatorname{Semistar}\left(D_{n}\right)\right|$ for all $n$, then $s_{n}=2^{\left(\begin{array}{c}m_{n} \\ \left.m_{n} / 2\right]\end{array}\right) f(n)}$, where $f(n) \geq 1$ for all $n$ and $f(n)=1+O\left(m_{n}^{-1 / 4} \log _{2} m_{n}\right)$ as $n \longrightarrow \infty$, and therefore $\log _{2} s_{n} \sim\left(\begin{array}{c}m_{n} \\ {\left[m_{n} / 2\right]}\end{array}\right)$ as $n \longrightarrow \infty$.

Note next that the poset $\left(2^{\mathcal{Z}(\operatorname{Max}(D))}\right)^{\dagger} \cong \operatorname{Semistar}(D)$ is constructed solely from the set $\operatorname{Max}(D)$. Therefore, if $\operatorname{Max}(D)$ and $\operatorname{Max}\left(D^{\prime}\right)$ have the same cardinality for two Dedekind domains $D$ and $D^{\prime}$, then the lattices Semistar $(D)$ and Semistar $\left(D^{\prime}\right)$ are isomorphic. Regarding the converse, at least we can say this. Consider the following statement.

(P) $\left|2^{X}\right|=\left|2^{Y}\right|$ implies $|X|=|Y|$ for all sets $X$ and $Y$.

It is well-known that ZFC $\forall \mathrm{P}$ (if ZFC is consistent) and ZFC+GCH $\vdash \mathrm{P}$, where ZFC is Zermelo-Fraenkel set theory with the Axiom of Choice, and GCH is the Generalized Continuum Hypothesis (which in ZFC is equivalent to the statement that $Y \subseteq 2^{X}$ implies $|Y| \leq|X|$ or $|Y|=\left|2^{X}\right|$ for all sets $X$ and $Y$ ). We have the following.

Corollary 4.6. Consider the following conditions on Dedekind domains D and $D^{\prime}$.

1. $\operatorname{Max}(D)$ and $\operatorname{Max}\left(D^{\prime}\right)$ have the same cardinality.

2. The near multiplicative lattices $\mathcal{K}(D)$ and $\mathcal{K}\left(D^{\prime}\right)$ are isomorphic.

3. The lattices $\operatorname{Semistar}(D)$ and $\operatorname{Semistar}\left(D^{\prime}\right)$ are isomorphic.

4. Semistar $(D)$ and $\operatorname{Semistar}\left(D^{\prime}\right)$ have the same cardinality.

One has $(1) \Leftrightarrow(2) \Rightarrow(3) \Rightarrow(4)$, and the four conditions are equivalent if either $\operatorname{Max}(D)$ or $\operatorname{Max}\left(D^{\prime}\right)$ is finite or if one assumes along with the axioms of ZFC the following statement: $(P)\left|2^{X}\right|=\left|2^{Y}\right|$ implies $|X|=|Y|$ for all sets $X$ and $Y$. Moreover, the implication (4) $\Rightarrow(1)$ is equivalent in $Z F C$ to the statement $(P)$ and therefore can neither be proved nor disproved from ZFC if ZFC is consistent.

Proof. By Proposition 3.1(4) and [3, Corollary 13.2] we have (1) $\Rightarrow(2) \Rightarrow$ $(3) \Rightarrow(4)$. Moreover, the elements of $\operatorname{Max}(D)$ can be recovered from the near multiplicative lattice $\mathcal{K}(D)$ as the elements lying immediately below the identity element $D$ of $\mathcal{K}(D)$. Thus we have (2) $\Rightarrow(1)$. Suppose, then, that condition (4) holds. If either $\operatorname{Max}(D)$ or $\operatorname{Max}\left(D^{\prime}\right)$ is finite, then from Corollary 1.2 it follows that $\operatorname{Max}(D)$ and $\operatorname{Max}\left(D^{\prime}\right)$ have the same (finite) cardinality. Alternatively, if $\operatorname{Max}(D)$ and $\operatorname{Max}\left(D^{\prime}\right)$ are infinite and one assumes the statement $(\mathrm{P})$, then since $2^{2^{|\operatorname{Max}(D)|}}=2^{2^{\left|\operatorname{Max}\left(D^{\prime}\right)\right|}}$ by Corollary 4.2 , one has $|\operatorname{Max}(D)|=\left|\operatorname{Max}\left(D^{\prime}\right)\right|$. Finally, since one can prove in ZFC that there is a PID $D$ with $|\operatorname{Max}(D)|$ equal to any given infinite cardinal $\aleph_{a}$ (namely, $D=k[T]$, where $k$ is an algebraic closure of $\mathbb{Q}(\mathbf{X})$, where $|\mathbf{X}|=\aleph_{a}$ ), it follows that the implication (4) $\Rightarrow(1)$ is equivalent in $\mathrm{ZFC}$ to the statement $(\mathrm{P})$ and therefore can neither be proved nor disproved from $\mathrm{ZFC}$ if $\mathrm{ZFC}$ is consistent.

Problem 4.7. In Corollary 4.6 does (3) imply (2)? Does (4) imply (3)? 


\section{The lattice of finite type semistar operations}

A semistar operation $\star$ on a domain $D$ is said to be of finite type if $I^{\star}=$ $\bigcup\left\{J^{*}: J \subseteq I\right.$ is finitely generated $\}$ for all $I \in \mathcal{K}(D)$. By [3. Proposition 13.6] the set $\operatorname{Semistar}_{f}(D)$ of all finite type star operations on $D$ is a sublattice of $\operatorname{Semistar}(D)$ that is closed under arbitrary suprema and finite infima; in particular it is a complete lattice. In this section we construct the lattice $\operatorname{Semistar}_{f}(D)$ for any Dedekind domain $D$. As one might suspect this is a much smaller lattice than $\operatorname{Semistar}(D)$ and is much easier to construct.

A closure operation $\star$ on a poset $S$ is said to be finitary if $(\bigvee \Delta)^{\star}=\bigvee\left(\Delta^{\star}\right)$ for all directed subsets $\Delta$ of $S$ for which $\bigvee \Delta$ exists. A semistar operation on a domain $D$ is of finite type if and only if it is finitary as a closure operation on the lattice $\mathcal{K}(D)$.

Let $M$ be a near multiplicative lattice. By [3, Proposition 5.3] the subset $\mathrm{N}_{f}(M)$ of $\mathrm{N}(M)$ consisting of all finitary nuclei on $M$ is closed under arbitrary suprema and in particular is a complete lattice. Let

$$
\mathrm{R}(M)=\left\{x \in M: x^{2}=x \text { and } x \geq 1\right\} .
$$

Then $\mathrm{R}(M)$ is also a near multiplicative lattice by [3, Proposition 9.1]. For any $a \in M$ we let $d_{a}: M \longrightarrow M$ be given by $x^{d_{a}}=a x$ for all $x \in M$. Then $d_{a}$ is a finitary nucleus on $M$, and by [3, Proposition 10.4] the map $d_{-}: \mathrm{R}(M) \longrightarrow$ $\mathrm{N}_{f}(M)$ is a supremum-preserving poset embedding.

Example 5.1. If $D$ is an integral domain, then $\mathrm{R}(\mathcal{K}(D))$ is the lattice $\mathcal{O}(D)$ of all overrings of $D$ and $\mathrm{N}_{f}(\mathcal{K}(D))$ is the lattice $\operatorname{Semistar}_{f}(D)$ of all finite type semistar operations on $D$. One has $I^{d_{A}}=A I$ for any overring $A$ of $D$ and any $I \in \mathcal{K}(D)$.

Proposition 5.2. Let $D$ be a Dedekind domain.

1. The map $\rho: 2^{\operatorname{Max}(D)} \longrightarrow \mathcal{O}(D)$ acting by $\rho: X \longmapsto \bigcap_{\mathfrak{p} \in X} D_{\mathfrak{p}}$ is an anti-isomorphism of posets.

2. The map $d_{-}: \mathcal{O}(D)=\mathrm{R}(\mathcal{K}(D)) \longrightarrow \operatorname{Semistar}_{f}(D)=\mathrm{N}_{f}(\mathcal{K}(D))$ is an isomorphism of posets.

3. The map $2^{\operatorname{Max}(D)} \longrightarrow \operatorname{Semistar}_{f}(D)$ acting by $X \longmapsto d_{\rho(X)}$ is an antiisomorphism of posets.

Proof. By [5, Proposition 3.14], if $A$ is an overring of a Dedekind domain $D$, then there exists a unique subset $X$ of $\operatorname{Max}(D)$ such that $A=\bigcap_{\mathfrak{p} \in X} D_{\mathfrak{p}}$. Statement (1) follows. Statement (2), then, follow from [3, Example 10.8], and statement (3) follows from statements (1) and (2).

Corollary 5.3. One has $\left|\operatorname{Semistar}_{f}(D)\right|=2^{|\operatorname{Max}(D)|}$ for any Dedekind domain $D$. 


\section{References}

[1] V. B. Alekseev, On the number of families of subsets closed with respect to intersections, Diskretnaya Matematika 1 (2) (1989) 129-136 (in Russian).

[2] P. Colomb, A. Irlande, O. Raynaud, Counting of Moore families for $n=7$, in: Formal Concept Analysis, Lecture Notes in Computer Science, Volume 5986, Springer Science+Business Media, Berlin, 2010, pp. 72-87.

[3] J. Elliott, Prequantales and applications to semistar operations and module systems, arxiv.org/abs/1101.2462.

[4] A. Higuchi, Lattice of closure operators, Discrete Math. 179 (1998) 267-272.

[5] W. Heinzer, M. Roitman, Well-centered overrings of an integral domain, J. Alg. 272 (2004) 435-455.

[6] W. Krull, Idealtheorie, Springer-Verlag, Berlin, 1935.

[7] R. Matsuda, Commutative Semigroup Rings, Second Edition, Kaisei, Tokyo, 2006.

[8] R. Matsuda, Note on valuation rings and semistar-operations, Comm. Algebra 28 (5) (2000) 2515-2519.

[9] A. Mimouni, Semistar-operations of finite character on integral domains, J. Pure Appl. Alg. 200 (2005) 37-50.

[10] S. B. Niefield, K. I. Rosenthal, Constructing locales from quantales, Math. Proc. Camb. Phil. Soc. 104 (2) (1988) 215-234.

[11] A. Okabe, R. Matsuda, Semistar-operations on integral domains, Math. J. Toyama Univ. 17 (1994) 1-21.

[12] G. Picozza, Star operations on overrings and semistar operations, Comm. Algebra 33 (2005) 2051-2073. 